\title{
Osteopathic Medical Care With and Without Osteopathic Manipulative Treatment in Patients With Chronic Low Back Pain: A Pain Registry-Based Study
}

John C. Licciardone, DO, MS, MBA; Robert J. Gatchel, PhD

From the Department of Family Medicine

(Dr Licciardone) and the Osteopathic Research Center (Drs Licciardone and Gatchel)

at the University of North Texas Health Science Center Texas College of Osteopathic Medicine in Fort Worth; and the Department of Psychology at the University of Texas at Arlington (Dr Gatchel).

Dr Licciardone is Regents Professor and the Osteopathic Heritage Foundation Richards-Cohen Distinguished Chair in Clinical Research. Dr Gatchel is the Nancy P. and John G. Penson Endowed Professor of Clinical Health Psychology and Distinguished Professor of Psychology.

Financial Disclosures: None reported.

Support: The study was supported in part by grants to

Dr Licciardone from the Osteopathic Heritage Foundation.

Address correspondence to John C. Licciardone, DO, MS, MBA, 3500 Camp Bowie Blvd, Fort Worth, TX 76107-2644.

E-mail:

john.licciardone@unthsc.edu

Submitted February 24, 2019; accepted April 3, 2019.
Context: The OSTEOPATHIC Trial demonstrated substantial improvement in pain intensity, decreased need for rescue medication for pain, and greater likelihood of recovery in patients with chronic low back pain who received 6 osteopathic manipulative treatment (OMT) sessions over 3 months.

Objective: To assess osteopathic medical care and the effectiveness of OMT for chronic low back pain in a real-world setting without the constraints of a rigid research protocol.

Methods: An observational study of 445 adults with chronic low back pain who had an established osteopathic physician (ie, DO) or allopathic physician (ie, MD) was conducted within the PRECISION Pain Research Registry from April 2016 through February 2019. Primary outcome measures included a numerical rating scale for low back pain intensity, the Roland-Morris Disability Questionnaire for back-related functioning, and use of nonsteroidal anti-inflammatory drugs or opioids for low back pain.

Results: A total of 79, 48, and 318 patients, respectively, were treated by DOs who used OMT, DOs who did not use OMT, or MDs. Patients treated by DOs who used OMT reported significantly lesser low back pain intensity (mean numerical rating score, 5.6; 95\% CI, 5.1-6.1 vs 6.1; 95\% CI, 5.9-6.3; $P=.04)$ and back-related disability (mean Roland-Morris Disability score, 12.4 ; 95\% CI, 11.1-13.8 vs 14.4; 95\% CI, $13.7-15.0 ; P=.009)$ than patients treated by MDs. Patients treated by DOs who used OMT also reported less frequent use of nonsteroidal anti-inflammatory drugs (multivariate odds ratio, $0.41 ; 95 \% \mathrm{CI}, 0.24-0.70 ; P=.001$ ) or opioids (multivariate odds ratio, $0.52 ; 95 \% \mathrm{CI}, 0.28-0.98 ; P=.04)$. There were no significant differences in primary outcomes between DOs who did not use OMT and MDs.

Conclusion: This study of community-based patients in a pain research registry supports the effectiveness of OMT as an integral component of osteopathic medical care for chronic low back pain. Patients treated by DOs who did not use OMT did not experience better results than patients treated by MDs in any primary outcome measure. Further research is needed to more specifically compare the effects of OMT with other treatment effects that may be attributed to patient-DO interactions during medical encounters.

J Am Osteopath Assoc. 2020;120(2):64-73

doi:10.7556/jaoa.2020.016

Keywords: low back pain, OMT, pain registry 
$\mathrm{R}$ andomized controlled trials, ${ }^{1-3}$ systematic reviews and meta-analyses, ${ }^{4,5}$ and national health care surveys ${ }^{6,7}$ in the United States provide varying levels of support for the view that osteopathic medical care, including osteopathic manipulative treatment (OMT), is a particularly effective intervention for low back pain. Based largely on findings from the first systematic review and meta-analysis conducted to assess this research question, ${ }^{4}$ the only clinical practice guideline developed by the American Osteopathic Association recommends using OMT in patients wherein somatic dysfunction is a cause of, or contributing factor to, low back pain. ${ }^{8}$

Specifically, with regard to chronic low back pain, the OSTEOPATHIC Trial has provided the most compelling evidence to date that supports the efficacy of OMT. $^{3,9}$ The OSTEOPATHIC Trial demonstrated substantial improvement in pain intensity, decreased need for rescue medication for pain, and greater likelihood of recovery in patients with chronic low back pain who received 6 OMT sessions during a 3-month period. ${ }^{3,9}$ Nevertheless, the extent to which such findings are generalizable to other patients with chronic low back pain remains unclear. The National Ambulatory Medical Care Survey (NAMCS) has been used in efforts to address this question. Therein, from 2003 to 2004 it was shown that osteopathic physicians (ie, DOs) provided a disproportionately large proportion of medical care visits for chronic low back pain. ${ }^{7}$ Osteopathic physicians also prescribed pharmacologic therapy less often and maintained greater continuity of care than allopathic physicians (ie, MDs) in another NAMCS study ${ }^{6}$ of patients with low back pain from 2002 to 2006 . However, the latter findings were not specific to chronic low back pain. Additionally, osteopathic research using NAMCS is limited in that it does not provide specific data on the use of OMT within patient visits. The purpose of the present study was to address such limitations and voids in the current osteopathic literature pertaining to the use of OMT for chronic low back pain in real-world settings by using data collected from a community-based pain research registry.

\section{Methods}

\section{Patient Recruitment, Screening, and}

\section{Enrollment}

Patients with chronic low back pain were recruited from the Pain Registry for Epidemiological, Clinical, and Interventional Studies and Innovation (PRECISION Pain Research Registry) within the Osteopathic Research Center at the University of North Texas Health Science Center Texas College of Osteopathic Medicine during the period of April 2016 through February 2019. The registry procedures and protocol for collecting patient-reported data within the Dallas-Fort Worth metroplex have been reported elsewhere. ${ }^{10}$ Registry staff screened patients for eligibility at multiple venues, including the group practice plan at the University of North Texas Health Science Center, local community events, and health fairs. Recruitment flyers were distributed at local hospitals, clinics, and physician offices to direct patients to the online screening questionnaire. Conference presentations, print advertising, emails, and letters were used to seek physician referrals for patient screening. Direct-to-patient advertising through the registry's website and social media were also used to screen patients. The research protocol was approved by the North Texas Regional Institutional Review Board (\#2015-169). All eligible patients provided written informed consent prior to enrolling in the registry and participating in this study.

\section{Inclusion and Exclusion Criteria}

The inclusion criteria involved having chronic low back pain according to the case definition established by the National Institutes of Health (NIH) Task Force on Research Standards for Chronic Low Back Pain. ${ }^{11}$ This criterion requires that patients self-report having low back pain for at least 3 to 6 months with a frequency of pain of at least half of the days during the past 6 months. Other inclusion criteria for this study were being 21 to 79 years of age; being able to respond to data collection instruments in English; and having a physician who had provided medical care for low back pain. Exclusion criteria were being pregnant (based on self-report) or being institutionalized. Patients received monetary compensation 
for their time and travel expenses to complete the baseline registry visit that included data for this study.

\section{Registry Data Collection}

The PRECISION Pain Research Registry routinely collects data to characterize patients and their clinical status upon enrollment. The relevant measures for this study are described in the following paragraphs.

\section{NIH Minimum Dataset for Low Back Pain}

This instrument is recommended by the NIH Task Force on Research Standards for Chronic Low Back Pain to describe patients participating in research studies. ${ }^{11}$ It consists of 40 patient-reported items, including sociodemographic characteristics, medical history, symptoms, functioning, and interventions for low back pain.

\section{SPADE Cluster for Quality of Life Deficits}

The 29-item Patient-Reported Outcomes Measurement Information System (PROMIS-29) is a quality of life measure that includes items derived from the PROMIS pain behavior item bank that assesses how low back pain interferes with normal activities and assesses levels of anxiety, depression, fatigue, and sleep disturbance. $^{12}$ The SPADE cluster (sleep disturbance, pain interference with activities, anxiety, depression, and low energy/fatigue scales) was used as a composite measure of quality of life, with higher scores representing greater quality of life deficits.

\section{History of Medical Conditions Inventory}

This inventory consists of a series of back-related or general medical conditions with which patients may report having ever been diagnosed. This study focused on herniated disc, sciatica, and depression, which are comorbidities commonly reported by patients with chronic low back pain.

\section{History of Nonpharmacologic Treatments for Low Back Pain Inventory}

This inventory was derived from a series of nonpharmacologic treatments evaluated in a systematic review $^{13}$ for the American College of Physicians Clinical Practice Guideline for low back pain. ${ }^{14}$ The present study focused on spinal manipulation as a nonpharmacologic treatment. The reported use of spinal manipulation for low back pain by patients currently treated by DOs was assumed to represent OMT.

\section{Primary Outcome Measures}

The primary outcome measures in this study included low back pain intensity, back-related functioning, and use of nonsteroidal anti-inflammatory drugs (NSAIDs) or opioids for low back pain. The numerical rating scale (NRS) for pain intensity and the Roland-Morris Disability Questionnaire (RMDQ) for back-related functioning are commonly used outcome measures for low back pain. The NRS is included in the NIH Minimum Dataset, and the NIH Task Force on Research Standards for Chronic Low Back Pain recommends the RMDQ as a legacy measure of back-related functioning. ${ }^{11}$ These 2 measures may also be combined to serve as a composite measure of recovery from chronic low back pain. ${ }^{15}$

Guidelines from the Centers for Disease Control and Prevention ${ }^{16}$ and the American College of Physicians ${ }^{14}$ both emphasize the need to initially select nonpharmacologic treatments for patients with chronic low back pain. Both guidelines recommend opioid therapy only if nonpharmacologic and first-line pharmacologic treatments such as NSAIDs fail to provide an adequate response and if the anticipated benefits of opioid therapy for a given patient outweigh the potential risks. ${ }^{14,16}$ Thus, the reported use of NSAIDs or opioids by patients with chronic low back pain may be taken as surrogate measures of physician adherence to recommended treatment guidelines.

\section{NRS for Low Back Pain Intensity}

The NRS used in this study measured low back pain "on average over the past 7 days" with an 11-point scale ranging from 0 ("no pain") to 10 ("worst pain").

\section{Roland-Morris Disability Questionnaire}

The RMDQ consists of 24 items that assess backrelated functioning by measuring how much low back 
pain adversely affects patient functioning and activities. ${ }^{17}$ Each item was scored as either 1 ("agree" that low back pain has an adverse impact) or 0 ("disagree" that low back pain has an adverse impact). The RMDQ is scored as the sum of responses to each item, thereby potentially ranging from 0 to 24 . The composite outcome of 1 or less on the NRS for low back pain intensity and 2 or less on the RMDQ may be taken to indicate recovery from chronic low back pain.

\section{Use of NSAIDs or Opioids for Low Back Pain}

The use of NSAIDs or opioids for low back pain was primarily assessed with an item from the NIH Minimum Dataset (for opioids) and an analogous item developed for reporting NSAID use. Such self-reported medication use has generally shown good agreement with pharmacy records. ${ }^{18}$

\section{Data Management and Statistical Analysis}

Patients completed the data collection instruments using a computer or mobile device provided by project personnel and the Qualtrics Survey Software. Research coordinators assisted patients if they had difficulty reading or understanding the questions. The electronic data collection system permitted patients to take breaks while answering questions if needed; however, it required completion of all items on the research instruments. Qualtrics survey data were exported to the SPSS version 23 software (IBM), which was subsequently used for data management and analysis. Descriptive statistics were computed using numbers and percentages for nominal and dichotomous variables and mean (SD) for continuous variables. Statistical comparisons of patients treated by physicians within each of the 3 categories (DOs who used OMT, DOs who did not use OMT, or MDs) were performed using contingency table methods for categorical variables or analysis of variance with the Fisher least significant difference test for post hoc comparisons for continuous variables. Additionally, analysis of covariance was performed to adjust the low back pain intensity and back-related functioning outcomes for patient age and sex. Simple logistic regression models were initially used to compute odds ratios (ORs) and 95\% CIs for patient-reported use of NSAIDs or opioids according to the type of treating physician, with MDs used as the reference category. Multiple logistic regression was subsequently used to adjust for potential confounders. Therein, the age, the NRS score for low back pain intensity, and the Roland-Morris Disability score were entered as covariates, and the ORs and 95\% CIs for use of the relevant drug category (NSAIDs or opioids) were adjusted for current use of the alternative drug category (opioids or NSAIDs). Hypotheses were tested at the .05 level of statistical significance.

\section{Results}

\section{Sociodemographic and Clinical}

\section{Characteristics of Patients}

There were 518 registry patients screened for study participation, and 445 (85.9\%) met the eligibility criteria (Figure 1). Patients ranged from 21 to 79 years of age, with a mean (SD) age of 54.0 (12.0) years. There were $308(69.2 \%)$ female patients. The mean (SD) low back pain intensity reported by patients was 6.1 (2.0) and the mean (SD) Roland-Morris Disability score was 14.1 (5.9). No patient met the composite pain and functioning criteria for recovery from chronic low back pain. A total of 288 patients $(64.7 \%)$ reported currently using NSAIDs for low back pain, 164 (36.9\%) reported using opioids, and 91 (20.4\%) reported using both NSAIDs and opioids. Osteopathic physicians treated 127 patients (28.5\%), including $79(17.8 \%)$ who reported receiving OMT for low back pain. There were no significant differences among patients at the time of registry enrollment by type of treating physician, with the exception that current smokers were less likely to be treated by DOs who used OMT (Table 1).

\section{Low Back Pain Intensity and Back-Related Disability}

There were significant differences in low back pain intensity reported by patients according to the type of treating physician $(P=.02)$ (Figure 2). Significant post 


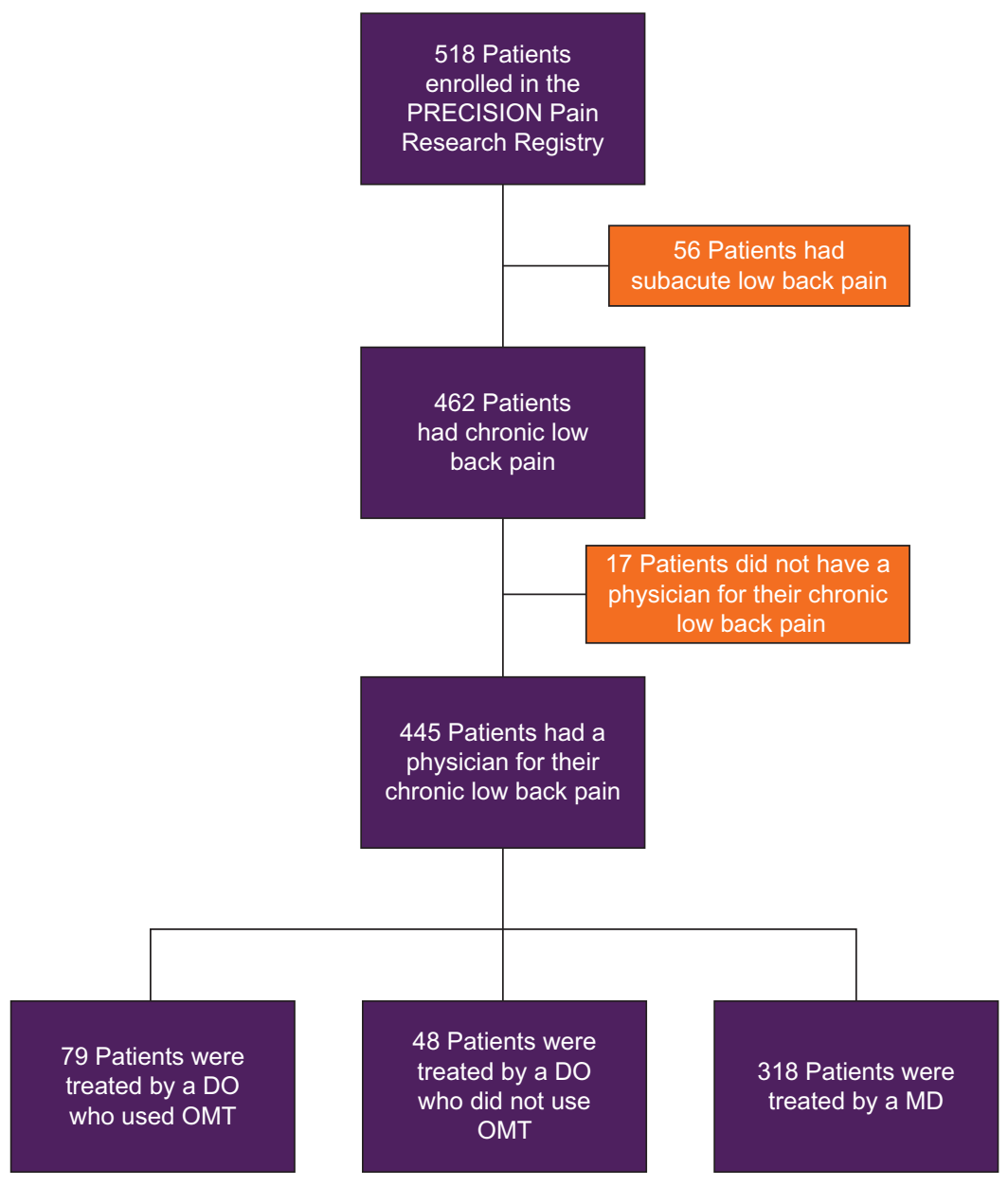

Figure 1.

Flowchart showing reasons for exclusion at each stage of the study and the distribution of patients according to type of physician. Abbreviations: DO, osteopathic physician; MD, allopathic physician; OMT, osteopathic manipulative treatment; PRECISION, Pain Registry for Epidemiological, Clinical, and Interventional Studies and Innovation.

hoc comparisons were observed for patients treated by DOs who used OMT vs patients treated by DOs who did not use OMT $(P=.008)$ and for patients treated by DOs who used OMT vs patients treated by MDs $(P=.04)$. Similarly, there were significant differences in back-related disability reported by patients according to the type of treating physician $(P=.02)$ (Figure 3). Significant post hoc comparisons were observed for patients treated by DOs who used OMT vs patients treated by DOs who did not use OMT $(P=.03)$ and for patients treated by DOs who used OMT vs patients treated by $\mathrm{MDs}(P=.009)$.

\section{Use of NSAIDs or Opioids for Low Back Pain}

Nonsteroidal anti-inflammatory drugs were reportedly used for low back pain by 41 (51.9\%), 30 (62.5\%), and $217(68.2 \%)$ patients treated by DOs who used OMT, DOs who did not use OMT, or MDs, respectively. The corresponding summary measures for NSAID use in comparison with patients treated by MDs were as follows: OR, 0.50; 95\% CI, 0.30-0.83; $P=.007$ for patients treated by DOs who used OMT; and OR, 0.78; 95\% CI, 0.41-1.46; $P=.43$ for patients treated by DOs who did not use OMT. Patients treated by DOs who used OMT also less frequently reported 
Table 1.

Sociodemographic and Clinical Characteristics of Patients With Chronic Low Back Pain by Type of Treating Physician $(\mathrm{N}=445)^{\mathrm{a}}$

\begin{tabular}{|c|c|c|c|c|}
\hline Characteristic & $\begin{array}{l}\text { DO Who Used } \\
\text { OMT }\end{array}$ & $\begin{array}{l}\text { DO Who Did Not } \\
\text { Use OMT }\end{array}$ & MD & $\begin{array}{c}P \\
\text { Value }\end{array}$ \\
\hline Total, $\mathrm{n}$ & 79 & 48 & 318 & \\
\hline Age, y, mean (SD) & $53.0(14.7)$ & $56.8(10.6)$ & $53.8(11.5)$ & .20 \\
\hline Female & $57(72.2)$ & $38(79.2)$ & $213(67)$ & .19 \\
\hline Current cigarette smoker & $7(8.9)$ & $12(25.0)$ & $71(22.3)$ & .02 \\
\hline SPADE quality of life score, ${ }^{b}$ mean (SD) & $10.4(3.2)$ & $11.1(3.2)$ & $10.9(3.1)$ & .37 \\
\hline Presence of widespread pain & $18(22.8)$ & $14(29.2)$ & $85(26.7)$ & .69 \\
\hline Diagnosis of herniated disc & $29(36.7)$ & $12(25.0)$ & $115(36.2)$ & .30 \\
\hline Diagnosis of sciatica & $31(39.2)$ & $14(29.2)$ & $104(32.7)$ & .44 \\
\hline Diagnosis of depression & $34(43.0)$ & $23(47.9)$ & $153(48.1)$ & .72 \\
\hline Previous low back surgery & $13(16.5)$ & $8(16.7)$ & $46(14.5)$ & .86 \\
\hline $\begin{array}{l}\text { Ever unemployed or unable to do usual work for } \geq 1 \text { month } \\
\text { due to low back pain }\end{array}$ & $27(34.2)$ & $14(29.2)$ & $133(41.8)$ & .15 \\
\hline $\begin{array}{l}\text { Ever received disability or workers' compensation benefits } \\
\text { because of inability to work due to low back pain }\end{array}$ & $16(20.3)$ & $11(22.9)$ & $82(25.8)$ & .57 \\
\hline $\begin{array}{l}\text { Ever involved in a lawsuit or legal claim related to low back } \\
\text { pain }\end{array}$ & $9(11.4)$ & $1(2.1)$ & $40(12.6)$ & .10 \\
\hline $\begin{array}{l}\text { Had current treating physician for low back pain for } 5 \text { years } \\
\text { or longer }\end{array}$ & $16(20.3)$ & $14(29.2)$ & $88(27.7)$ & .37 \\
\hline
\end{tabular}

a Data are given as No. (\%) unless otherwise indicated.

b Higher scores on the SPADE cluster represent poorer quality of life.

Abbreviations: DO, osteopathic physician; MD, allopathic physician; NSAID, nonsteroidal anti-inflammatory drug; OMT, osteopathic manipulative treatment; SPADE, sleep disturbance, pain interference with activities, anxiety, depression, low energy/fatigue.

using NSAIDs in the multivariate analysis that controlled for potential confounders (OR, 0.41; 95\% CI, 0.24-0.70; $P=.001$ ) (Table 2). Increasing age, being a male patient, having a diagnosis of a herniated disc, and currently using opioids were other patient factors significantly associated with reporting less frequent use of NSAIDs for low back pain.

Opioids were reportedly used for low back pain by 20 (25.3\%), 17 (35.4\%), and 127 (39.9\%) patients treated by DOs who used OMT, DOs who did not use OMT, or MDs, respectively. The corresponding summary measures for opioid use in comparison with patients treated by MDs were as follows: OR, 0.51;
95\% CI, $0.29-0.89 ; P=.02$ for patients treated by DOs who used OMT; and OR, 0.82; 95\% CI, 0.44-1.55 $P=.55$ for patients treated by DOs who did not use OMT. Patients treated by DOs who used OMT also less frequently reported using opioids in the multivariate analysis (OR, 0.52; 95\% CI, 0.28-0.98; $P=.04$ ). Current cigarette smoking, presence of widespread pain, having a diagnosis of a herniated disc, and increasing levels of back-related disability were patient factors significantly associated with reporting more frequent use of opioids for low back pain, whereas reported current use of NSAIDs was associated with less frequent use of opioids. 


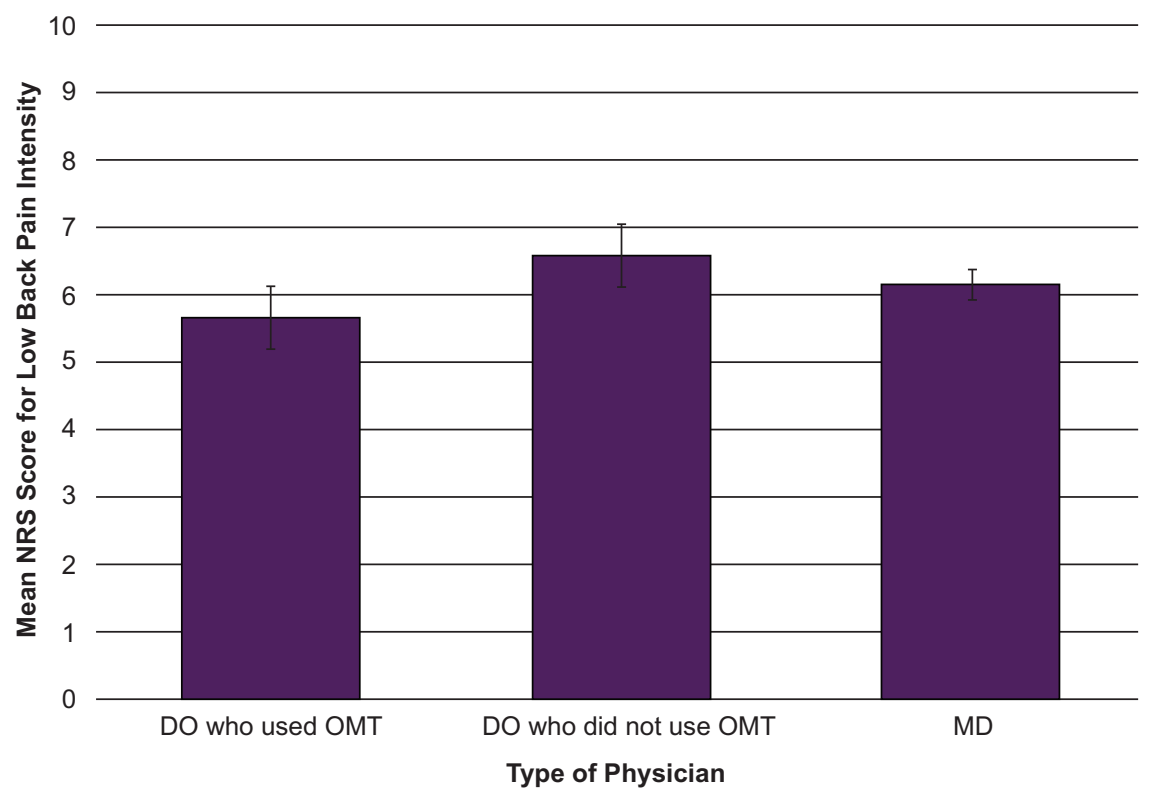

Figure 2.

Patient-reported measures of low back pain intensity at the time of enrollment in the PRECISION Pain Research Registry by type of physician $(P=.02)$. Low back pain intensity was measured with a numerical rating scale (NRS). The error bars represent $95 \% \mathrm{Cls}$. Significant post hoc comparisons were observed for osteopathic physicians (DOs) who used osteopathic manipulative treatment (OMT) vs DOs who did not use OMT $(P=.008)$ and for DOs who used OMT vs allopathic physicians (MDs) $(P=.04)$.

\section{Discussion}

This study involving a community-based pain research registry supports the effectiveness of OMT as an integral component of osteopathic medical care for chronic low back pain. The study findings further suggest that a standardized algorithmic approach to OMT, which is desirable in explanatory clinical trials, does not need to be used to achieve clinically relevant outcomes in real-world settings. Previous research in the OSTEOPATHIC Trial has shown that well-defined subgroups of patients with chronic low back pain may be identified and targeted for OMT based on their low back pain intensity and backrelated functioning. ${ }^{19}$ The present findings now show that OMT may be effectively individualized or "tailored" for a wide variety of patients with chronic low back pain. Clinical practice guidelines from the Centers for Disease Control and Prevention ${ }^{16}$ and the American College of Physicians ${ }^{14}$ emphasize the importance of initiating management for chronic low back pain with nonpharmacologic treatments and avoiding opioid therapy unless the benefits outweigh the risks for a given patient The present study indicates that patients treated by DOs who used OMT as a nonpharmacologic treatment were less likely to use NSAIDs or opioids for their low back pain. Patients treated by DOs who did not use OMT did not experience better results than patients treated by MDs in any primary outcome measure. Thus, it appears that using OMT as a component of osteopathic medical care for chronic low back pain facilitates the successful implementation of current clinical practice guidelines.

Of the 127 patients treated by DOs, 79 (62.2\%) reported having received OMT. It is unclear why more than one-third of patients treated by DOs never received OMT. Two possible explanations are that some patients did not wish to receive OMT or that their physicians did not provide OMT for various reasons. One reason may have been that patients did not have somatic dysfunction that was the cause of, or a contributing factor to, their chronic low back pain. ${ }^{8}$ Other reasons may have included 


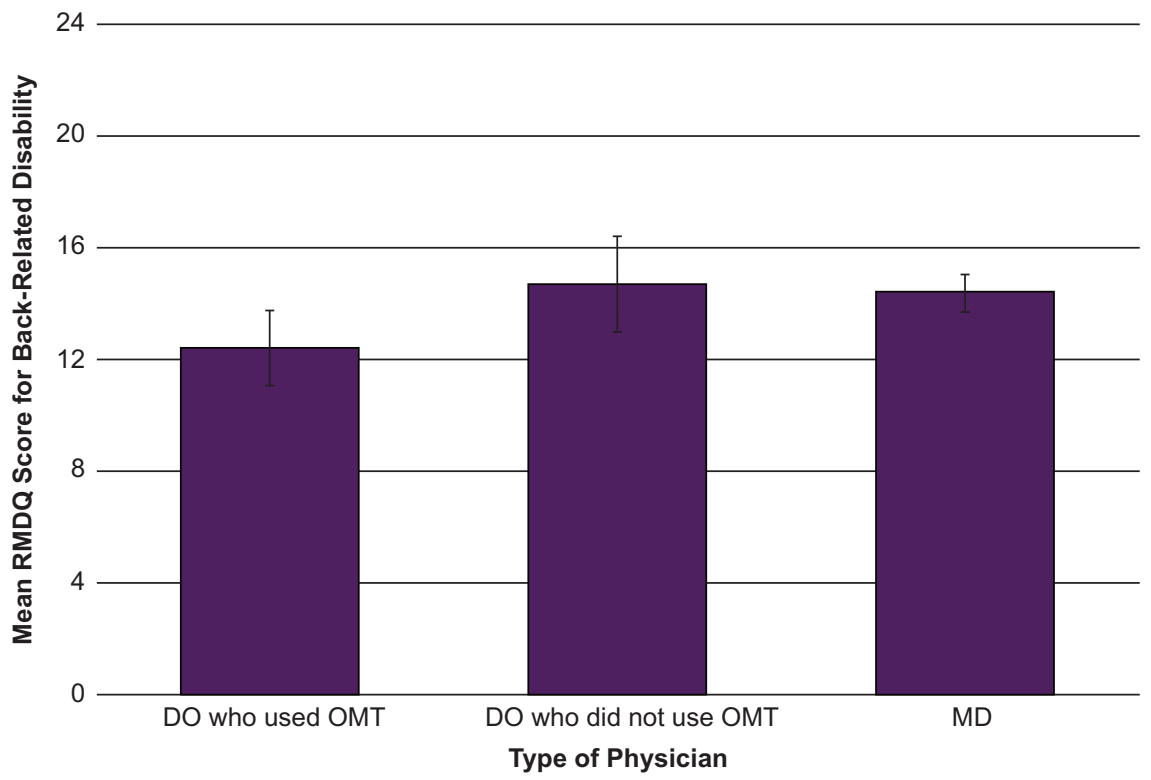

Figure 3.

Patient-reported measures of back-related disability at the time of registry enrollment by type of physician $(P=.02)$. Back-related disability was measured with the Roland-Morris Disability Questionnaire (RMDQ). The error bars represent 95\% Cls. Significant post hoc comparisons were observed for osteopathic physicians (DOs) who used osteopathic manipulative treatment (OMT) vs DOs who did not use OMT $(P=.03)$, and for DOs who used OMT vs allopathic physicians (MDs) $(P=.009)$.

insufficient time within the medical encounter to provide OMT, limited insurance coverage or inadequate reimbursement for OMT, or lack of physician comfort or confidence in providing OMT. There were no apparent patient sociodemographic or clinical characteristics that adequately explained the lack of OMT among 48 patients treated by DOs (Table 1).

There were several strengths of our study. Patients were recruited from a community-based pain research registry, thereby enabling us to study osteopathic medical care and use of OMT in a representative sample of patients with chronic low back pain. Recommendations of the NIH Task Force on Research Standards for Chronic Low Back Pain were adopted in conducting our study, including use of the case definition for chronic low back pain, recommended primary outcome measures pertaining to low back pain intensity and back-related functioning, and use of opioid therapy for low back pain. ${ }^{11}$ The greatest limitation of the study was its lack of randomization. However, this limitation did not appear to be an import- ant source of bias, as patients within each of the 3 study groups were not significantly different from one another on any sociodemographic or clinical characteristic, except cigarette smoking status (Table 1). Moreover, multivariate analyses that adjusted the primary outcomes for potential confounders did not materially affect the interpretation of the initially observed results.

Further research is needed to more specifically compare the OMT effects within osteopathic medical care for chronic low back pain with other effects that may be attributed to patient-physician interactions during medical encounters. Such factors as physician interpersonal manner, empathy, and communication style have been hypothesized to represent characteristics of DOs that may yield more favorable clinical outcomes among their patients, independent of the use of OMT. A formal mediation analysis that simultaneously considers the role of such patient-physician interactions and use of OMT is an important next step in elucidating the effects of osteopathic medical care for chronic low back pain. 
Table 2.

Multiple Logistic Regression for Factors Associated With Current Use of Nonsteroidal Anti-Inflammatory Drugs or Opioids for Chronic Low Back Pain $(\mathrm{N}=445)^{a}$

\begin{tabular}{|c|c|c|c|c|}
\hline \multirow{4}{*}{$\begin{array}{l}\text { Characteristic } \\
\text { Type of Physician }\end{array}$} & \multicolumn{4}{|c|}{ Current User } \\
\hline & \multicolumn{2}{|c|}{ NSAIDs } & \multicolumn{2}{|c|}{ Opioids } \\
\hline & OR (95\% Cl) & $P$ Value & OR $(95 \% \mathrm{Cl})$ & $P$ Value \\
\hline & & & & \\
\hline $\mathrm{MD}$ & 1.00 & $\ldots$ & 1.00 & $\ldots$ \\
\hline DO who used OMT & $0.41(0.24-0.70)$ & .001 & $0.52(0.28-0.98)$ & .04 \\
\hline DO who did not use OMT & $0.70(0.36-1.36)$ & .30 & $0.79(0.38-1.63)$ & .52 \\
\hline Age & $0.98(0.96-1.00)$ & .047 & $1.01(0.99-1.03)$ & .24 \\
\hline \multicolumn{5}{|l|}{ Sex } \\
\hline Female & 1.00 & $\ldots$ & 1.00 & $\ldots$ \\
\hline Male & $0.62(0.40-0.97)$ & .04 & $0.90(0.55-1.48)$ & .69 \\
\hline \multicolumn{5}{|l|}{ Cigarette Smoking Status } \\
\hline Never or Former Smoker & 1.00 & $\ldots$ & 1.00 & $\ldots$ \\
\hline Current Smoker & $0.91(0.52-1.57)$ & .72 & $2.13(1.21-3.75)$ & .009 \\
\hline \multicolumn{5}{|l|}{ Presence of Widespread Pain } \\
\hline No & 1.00 & $\ldots$ & 1.00 & $\ldots$ \\
\hline Yes & $0.87(0.53-1.44)$ & .60 & $1.81(1.09-3.00)$ & .02 \\
\hline \multicolumn{5}{|l|}{ Diagnosis of a Herniated Disc } \\
\hline No & 1.00 & $\ldots$ & 1.00 & $\ldots$ \\
\hline Yes & $0.55(0.35-0.87)$ & .01 & $2.45(1.54-3.91)$ & $<.001$ \\
\hline \multicolumn{5}{|l|}{ Diagnosis of Sciatica } \\
\hline No & 1.00 & $\ldots$ & 1.00 & $\cdots$ \\
\hline Yes & $1.43(0.91-2.24)$ & .12 & $1.41(0.88-2.24)$ & .15 \\
\hline \multicolumn{5}{|l|}{ Diagnosis of Depression } \\
\hline No & 1.00 & $\ldots$ & 1.00 & $\ldots$ \\
\hline Yes & $1.18(0.76-1.83)$ & .46 & $1.20(0.76-1.90)$ & .44 \\
\hline Low Back Pain Intensity & $0.97(0.86-1.08)$ & .56 & $0.95(0.84-1.08)$ & .41 \\
\hline Back-Related Disability & $1.01(0.97-1.06)$ & .56 & $1.10(1.05-1.15)$ & $<.001$ \\
\hline \multicolumn{5}{|l|}{ Currently Uses NSAID } \\
\hline No & $\cdots$ & $\ldots$ & 1.00 & $\ldots$ \\
\hline Yes & $\ldots$ & $\ldots$ & $0.55(0.34-0.87)$ & .01 \\
\hline \multicolumn{5}{|l|}{ Currently Uses Opioids } \\
\hline No & 1.00 & $\ldots$ & $\ldots$ & $\ldots$ \\
\hline Yes & $0.55(0.35-0.87)$ & .01 & $\ldots$ & $\ldots$ \\
\hline
\end{tabular}

a Odds ratios (ORs) and 95\% Cls were computed using multiple logistic regression for current use of nonsteroidal anti-inflammatory drugs (NSAIDs) or opioids. Age, low back pain intensity, and back-related disability were entered as covariates in these models, with the ORs and $95 \%$ Cls for these variables representing each unit increase in age $(y)$, numerical rating scale score, and Roland-Morris Disability score, respectively.

Abbreviations: DO, osteopathic physician; MD, allopathic physician; OMT, osteopathic manipulative treatment. 


\section{Conclusion}

This study of community-based patients in a pain research registry supports the effectiveness of OMT as an integral component of osteopathic medical care for chronic low back pain. Patients treated by DOs who used OMT experienced superior clinical outcomes pertaining to low back pain intensity and back-related functioning, while less frequently using NSAIDs or opioids, than patients treated by MDs. Patients treated by DOs who did not use OMT did not experience such outcomes. Further research is needed to more specifically compare the effects of OMT with the other effects of osteopathic medical care that may be attributed to patient-physician interactions during medical encounters.

\section{Acknowledgments}

We thank the staff of the PRECISION Pain Research Registry and the patients enrolled in the registry for their contributions to this study.

\section{Author Contributions}

Both authors provided substantial contributions to conception and design, acquisition of data, or analysis and interpretation of data; both authors drafted the article or revised it critically for important intellectual content; both authors gave final approval of the version of the article to be published; and both authors agree to be accountable for all aspects of the work in ensuring that questions related to the accuracy or integrity of any part of the work are appropriately investigated and resolved.

\section{References}

1. Hoehler FK, Tobis JS, Buerger AA. Spinal manipulation for low back pain. JAMA. 1981;245(18):1835-1838.

2. Andersson GB, Lucente T, Davis AM, Kappler RE, Lipton JA, Leurgans S. A comparison of osteopathic spinal manipulation with standard care for patients with low back pain. New Engl J Med. 1999;341(19):1426-1431. doi:10.1056/NEJM199911043411903

3. Licciardone JC, Minotti DE, Gatchel RJ, Kearns CM, Singh KP. Osteopathic manual treatment and ultrasound therapy for chronic low back pain: a randomized controlled trial. Ann Fam Med. 2013;11(2):122-129. doi:10.1370/afm.1468

4. Licciardone JC, Brimhall AK, King LN. Osteopathic manipulative treatment for low back pain: a systematic review and meta-analysis of randomized controlled trials. BMC Musculoskelet Disord. 2005;6:43. doi:10.1186/1471-2474-6-43

5. Franke H, Franke JD, Fryer G. Osteopathic manipulative treatment for nonspecific low back pain: a systematic review and meta-analysis. BMC Musculoskelet Disord. 2014;15:286. doi:10.1186/ 1471-2474-15-286
6. Licciardone JC. A national study of primary care provided by osteopathic physicians. J Am Osteopath Assoc. 2015;115 (12):704-713. doi:10.7556/jaoa.2015.145

7. Licciardone JC. The epidemiology and medical management of low back pain during ambulatory medical care visits in the United States. Osteopath Med Prim Care. 2008;2(1):11. doi:10.1186/ $1750-4732-2-11$

8. Clinical Guideline Subcommittee on Low Back Pain. American Osteopathic Association guidelines for osteopathic manipulative treatment (OMT) for patients with low back pain. J Am Osteopath Assoc. 2010;110(11):653-666. doi:10.7556/jaoa.2016.107

9. Licciardone JC, Gatchel RJ, Aryal S. Recovery from chronic low back pain after osteopathic manipulative treatment: a randomized controlled trial. J Am Osteopath Assoc. 2016;116(3):144-155. doi:10.7556/ jaoa.2016.031

10. Licciardone JC, Gatchel RJ, Phillips N, Aryal S. The Pain Registry for Epidemiological, Clinical, and Interventional Studies and Innovation (PRECISION): registry overview and protocol for a propensity score-matched study of opioid prescribing in patients with low back pain. J Pain Res. 2018;11:1751-1760. doi:10.2147/JPR.S169275

11. Deyo RA, Dworkin SF, Amtmann D, et al. Report of the NIH Task Force on research standards for chronic low back pain. J Pain. 2014;15(6):569-585. doi:10.1016/j.jpain.2014.03.005

12. Revicki DA, Chen WH, Harnam N, et al. Development and psychometric analysis of the PROMIS pain behavior item bank. Pain. 2009;146(1-2):158-169. doi:10.1016/j.pain.2009.07.029

13. Chou R, Deyo R, Friedly J, et al. Nonpharmacologic therapies for low back pain: a systematic review for an American College of Physicians clinical practice guideline. Ann Intern Med. 2017;166(7):493-505. doi:10.7326/M16-2459

14. Qaseem A, Wilt TJ, McLean RM, Forciea MA, Clinical Guidelines Committee of the American College of Physicians. Noninvasive treatments for acute, subacute, and chronic low back pain: a clinical practice guideline from the American College of Physicians. Ann Intern Med. 2017;166(7):514-530. doi:10.7326/M16-2367

15. Kamper SJ, Maher CG, Herbert RD, Hancock MJ, Hush JM, Smeets RJ. How little pain and disability do patients with low back pain have to experience to feel that they have recovered? Eur Spine J. 2010;19 (9):1495-1501. doi:10.1007/s00586-010-1366-1

16. Dowell D, Haegerich TM, Chou R. CDC guideline for prescribing opioids for chronic pain-United States, 2016. MMWR Recomm Rep. 2016;65(No. RR-1):1-49. doi:10.15585/mmwr.rr6501e1

17. Roland M, Morris R. A study of the natural history of back pain. Part I: development of a reliable and sensitive measure of disability in low-back pain. Spine. 1983;8(2):141-144. doi:10.1097/ 00007632-198303000-00004

18. Drieling RL, LaCroix AZ, Beresford SA, Boudreau DM, Kooperberg C, Heckbert SR. Validity of self-reported medication use compared with pharmacy records in a cohort of older women: findings from the Women's Health Initiative. Am J Epidemiol. 2016;184(3):233-238. doi:10.1093/aje/kwv446

19. Licciardone JC, Gatchel RJ, Aryal S. Targeting patient subgroups with chronic low back pain for osteopathic manipulative treatment: responder analyses from a randomized controlled trial. $J \mathrm{Am}$ Osteopath Assoc. 2016;116(3):156-168. doi:10.7556/jaoa.2016.032

๑) 2020 American Osteopathic Association 